\title{
Academic Ability And Teamworking In Medical Students
}

Do High Achieving Students Work Well As A Team?

\section{Authors:}

Parker R, Hodierne L, Anderson E, Davies RSM, Elloy M

\section{Correspondence:}

Dr. R Parker

Clinical Teaching Fellow

University of Leicester,

Centre for Medicine,

University Road,

Leicester,

LE1 7RH,

United Kingdom

Email: rupert.parker@gmail.com

Tel: 07764770446 


\begin{abstract}
Background

Teamworking is an essential skill for a doctor to develop in order to work effectively, and is required in the UK as part of the General Medical Council (GMC) Good Medical Practice guidance. Assessment of teamwork may be difficult, however, with medical school assessments being more commonly focused on knowledge and individual skills. We aim to explore the link between academic ability as measured at final medical examinations and teamworking.
\end{abstract}

\title{
Methods
}

All final-year medical students were asked to attend a simulation session in an immersive 22-bed simulated ward, which used a combination of patient simulators and high-fidelity manikin simulators, with nursing and telephone support. Students were split into separate groups stratified by performance in final-year assessments or in groups with mixed performance. Students were observed in real time by faculty staff and assessed with the individual Teamwork Observation and Feedback Tool (iTOFT), around which the debriefing was centred.

\section{Results}

The performance of 119 students in simulation was assessed, and groups with a mix of academic performance showed significantly greater teamworking ability as measured with the iTOFT as compared with those stratified by performance $(p=0.045)$. Final assessment at medical school was shown to be a poor predictor of teamworking ability: those who performed best at assessment seemed to underperform in teamworking.

\section{Discussion}


The simulated-ward learning activity received positive feedback, although the mix of patient simulators and highfidelity manikins proved a challenge to some students.

Medical school assessments appear to be inadequate in the assessment of teamworking ability, with change needed in future to ensure that this and other non-technical skills are assessed. 


\section{Introduction}

In the United Kingdom, final year medical students apply for positions in the Foundation Programme competitively. Students are ranked in a number of areas with the largest weighting on the Situational Judgement Test (1). This is a written examination undertaken during which students report what they consider to be the most appropriate action to take when faced with a set of hypothetical scenarios. There is evidence that Situational Judgement Tests predict behaviour in the workplace more reliably than interviews or their predecessor, the 'white space' question (2).

The ability of a doctor to work effectively in a team is an important skill component of a competent practitioner; teamworking is a specific competence within the GMC guidance: Good Medical Practice (3). Within postgraduate education, teamworking skills have been a cornerstone of simulation based learning and increasingly medical schools are including elements of teamwork skills teaching within their curricula(4). In contrast to knowledge based outcomes that are traditionally assessed within medical school examinations, the skills that allow an individual to perform competently within a team prove a more novel challenge for assessment, and in the main attempts to do so have focussed on surrogate markers such as self-reported attitudinal change and related knowledge recall (5). At the point of exit from medical school, most UK graduates are only formally assessed on the attributes that make them able to work in a team via the Situational Judgement Test, which is based upon how a student reports they would act rather than a direct assessment of their behaviour.

Within the postgraduate education domain there exists several educational tools to directly assess team working ability both as a function of the team as a whole such as the Team Emergency Assessment Measure (6), or as a measure of observable 
behaviours in individuals that contribute to effective teamwork for example the Anaesthetists Non-Technical Skills System (7). However, they are rarely transferrable to the undergraduate setting due to them being designed to assess within either a narrow clinical domain or being specialty specific. To address the lack of an appropriate undergraduate assessment tool the individual Teamwork Observation and Feedback Tool (iTOFT) (8) has been developed to measure observable team working behaviours in individuals working in small clinical teams in an attempt to provide a concrete framework for assessment of student team working ability. The tool consists of 10 desirable behaviour descriptors and a simple 4-point scale that is used to score appropriateness of the behaviour observed in the scenario assessed.

We report a novel study utilising the iTOFT to explore and assess the relationship between an individual's teamworking ability during an immersive ward based simulation and their performance in medical school final examinations. 


\section{Methods}

\section{Study design}

This was a double-blinded, prospective cohort study conducted with final year medical students.

\section{Simulation design}

As part of the medical school's 'preparation for practice' course (a two week course run at the end of final year designed to ease the transition from medical school to the workplace; all final year medical students who had passed their final assessment were invited to attend one of twelve simulation based training (SBT) session. The aim of the session was to assess a students' teamworking abilities whilst working within a small clinical team (5-6 students) within a 22 bed fully immersive simulated ward. Tasks were set in line with the UK Foundation Programme Curriculum to reflect the standard expected of a newly-qualified doctor (for example venepuncture, prescribing tasks or communication with colleagues). These tasks were presented to students using patient simulators and mixed-fidelity human patient simulators, with experienced on-site nursing support and senior advice over the telephone. In each of the 12 sessions students were split into 3 clinical teams.

Sessions commenced with a consultant led handover at which the students were given a 'job list' for patients they would be caring for with the students tasked with prioritising and distributing those jobs. There were some patients whose care was shared between the groups to simulate real world hospital shared-care arrangements. During the course of the simulation additional tasks were provided to the students including phone calls 
from relatives, reacting to abnormal pathology results and being asked to review deteriorating patients.

The student groups were selected by performance in final year assessments after being stratified into thirds: high-, mid- and low-achieving. These were then randomised into the 2 study groups: stratified and mixed ability. The groups stratified by final assessment scores were further stratified by thirds creating 4 groups in total (high achieving, mid achieving, low achieving and mixed ability). Groups were preselected by an external member of staff with the researchers and faculty being blinded to the selection process.

Students were observed in real-time by faculty members and scored using a modified basic version of iTOFT (10) (Table 1). Faculty members marked whether the students demonstrated each of the 13 attributes described in iTOFT or whether this was only partially met. After 40 minutes the simulation was brought to an end and the students were debriefed; comprehensive feedback was centred around iTOFT.

\section{Analysis}

Data were analysed using SPSS 22.0 and were non-normal in distribution (ShapiroWilk test) with the Kruskall Wallis test used for multiple groups and the Mann-Whitney U test used for direct comparison of median scores. Data are described according to median(IQR).

Where there were missing items scores were averaged and multiplied to produce a score out of 13 . Where a student had only partially met a domain they were scored a half point. 


\section{Ethics}

The study was approved by the University of Leicester Ethics Sub-Committee for Medicine and Biological Sciences. 


\section{Results}

\section{Student groups}

225 students were invited to the simulation, of these $184(82 \%)$ attended and $165(90 \%)$ consented to study enrolement. Of the 165 students, 46(28\%) were removed from analysis as there was inadequate data collected; this was either due to lost forms (one faculty member distributed the forms to the students to take home and were irretrievable) or faculty had completed the iTOFT incorrectly (figure 1). As a result 119 data sets were analysed with 60 students from mixed groups and 59 from stratified (Table 2).

\section{Teamworking ability}

Initial analysis of iTOFT scores showed a non-parametric distribution with left skew with the majority of our students demonstrating good team working abilities.

Those students who were in mixed ability groups had a median (IQR) iTOFT score of 11.2(3.5) which was greater than those assigned to the stratified groups who had a median(IQR) score of 10.5(1.625) (p:0.045). Of the stratified group no significant difference was seen between any of the subgroups median(IQR) 9.2(2.79) top, 11.2(1.55) middle, 10.5(0.5) (p:0.549) (see Table 2). 


\section{Discussion}

Our study found that final year medical school assessment results are a poor predictor of teamwork ability; our highest performing third of students didn't show any superior teamworking ability. Considering that the teamworking ability of a doctor is an important part of their competency (3), the analysis of data from this project raises questions about whether finals assessments are currently failing to adequately assess graduating medical students in this area. This suggests that our medical school assessments are not assessing all of what is required of a junior doctor particularly when it comes to teamworking ability and perhaps across other non-technical skills. Our data suggests that the SJT is not up to task in assessing desirable non-technical skills, such as teamworking, and a different combination of assessments may be required by hospitals and medical recruiters who have a large number of applicants. Current medical school results have only moderate predictive value in performance in future careers (9) and perhaps more focus could be spent on assessing teamworking ability to enhance predictive validity.

The transitions from distressed standardised patients to simulators showing signs of critical illness provided some difficulty for our students and this was reflected in low scores in the items assessing how the students put forwards patient's rights or integrating patient's understanding. Student and faculty feedback revealed a preference for standardised patients over simulators, a view which is supported in the literature (10). 
Our study was unable to detect any significant difference in teamworking ability between high and low performing students, this may be because of the limited number of participants or may could be a characteristic of the cohort assessed. Future work could address this by repeat the simulation across different years.

\section{iTOFT}

Our initial experience of the iTOFT tool is that faculty members were able to pick out a range of different behaviours across the students and reported that the tool allowed them to differentiate between students with excellent teamworking skills and those without.

However it appears a difficult tool to use when assessing whole teams, we found that faculty members often struggled to rate 5 to 6 students each on a 13 item assessment tool. $28 \%$ of iTOFT forms were so incomplete that the students were not rated in any domain, and of the remaining iTOFT forms analysed only $60(50 \%)$ were fully completed with the student scored on every domain. This is likely due to a number of factors, but ultimately requiring a single faculty member to score 5-6 students on a 13 item scale after each simulation was challenging and proved unreasonable. To mitigate this effect in future it would be beneficial to arrange greater faculty training on the iTOFT, and to have a better faculty to student ratio. In this simulation the iTOFT was used as the basis for debrief, however the use of a video recording system would enable researchers to assess the students retrospectively, faculty would continue to debrief with their preferred model.

\section{Future research}

We suggest that comparing teamwork assessments at the exit point from medical school with career performance in subsequent years and in different training environments may 
provide some insight into which students may go on to struggle in the workplace, which could help to guide career and workforce planning strategies. 


\section{References}

1. The Foundation Programme. Foundation Programme/Academic Foundation Programme Applicants’ Handbook. 2016 Jun 15.

2. Patterson F, Ashworth V, Zibarras L, Coan P, Kerrin M, O’Neill P. Evaluations of situational judgement tests to assess non-academic attributes in selection. Medical Education. 2012 Sep 1;46(9):850-68.

3. General Medical Council. Good Medical Practice. 2013 Mar 25.

4. Lerner S, Magrane D, Friedman E. Teaching teamwork in medical education. Mount Sinai Journal of Medicine: A Journal of Translational and Personalized Medicine. 2009 Aug 1;76(4):318-29.

5. Banerjee A, Slagle JM, Mercaldo ND, Booker R, Miller A, France DJ, Rawn L, Weinger MB. A simulation-based curriculum to introduce key teamwork principles to entering medical students. BioMed Central Medical Education. 2016 Nov 16;16(1):295.

6. Cooper S, Cant R, Porter J, Sellick K, Somers G, Kinsman L, et al. Rating medical emergency teamwork performance: Development of the Team Emergency Assessment Measure (TEAM). Resuscitation. 2010;81(4):446-52.

7. Fletcher G, Flin R, McGeorge P, Glavin R, Maran N, Patey R. Anaesthetists' Non-Technical Skills (ANTS): evaluation of a behavioural marker system. British Journal of Anaesthesia. 2003 May 1;90(5):580-8.

8. Thistlethwaite J, Dallest K, Moran M, Dunston R, Roberts C, Eley D, Bogossian F, Forman D, Bainbridge L, Drynan D, Fyfe S. Introducing the individual Teamwork Observation and Feedback Tool (iTOFT): Development and 
description of a new interprofessional teamwork measure. Journal of Interprofessional Care. 2016 Jul 3;30(4):526-8.

9. Hamdy H, Prasad K, Anderson MB, Scherpbier A, Williams R, Zwierstra R, Cuddihy H. Best Evidence Meducal Education systematic review: predictive values of measurements obtained in medical schools and future performance in medical practice. Medical Teacher. 2006 Jan 1;28(2):103-16.

10. May W, Park JH, Lee JP. A ten-year review of the literature on the use of standardized patients in teaching and learning: 1996-2005. Medical Teacher. 2009 Jan 1;31(6):487-92. 


\section{Tables \& Figures}

THE BEGINNING OF THE SIMULATION

Average score

Do the students form a team at the onset of the simulation?

DURING THE SIMULATION

\section{Shared Decision Making, Descriptor for scoring}

How students engage with the team actively engage with the

0.90

team.

How the students prioritise actions in care management.

0.78

How the students engage to review the goals of the care

0.71

management plan.

How the students engage to put a case for the rights of the

0.68

patient.

How students exchange information.

How students integrate patients understandings into the groups

0.70

management plans.

How the student recognises the boundaries of his her scope of practice.

\section{Working in a Team, Descriptor for scoring}

How the student participates in interprofessional discussions.

0.87

How the students demonstrates respect for others.

0.88

How the students actively seeks information from other team

0.82

members.

How students actively engage in discussion about the team

0.86 performance. 


\section{THE END OF THE SIMULATION}

Do the students reflect on their practice at the end of the

0.85

simulation?

Table 1: Scoring items from the modified iTOFT assessment tool and their

performance 


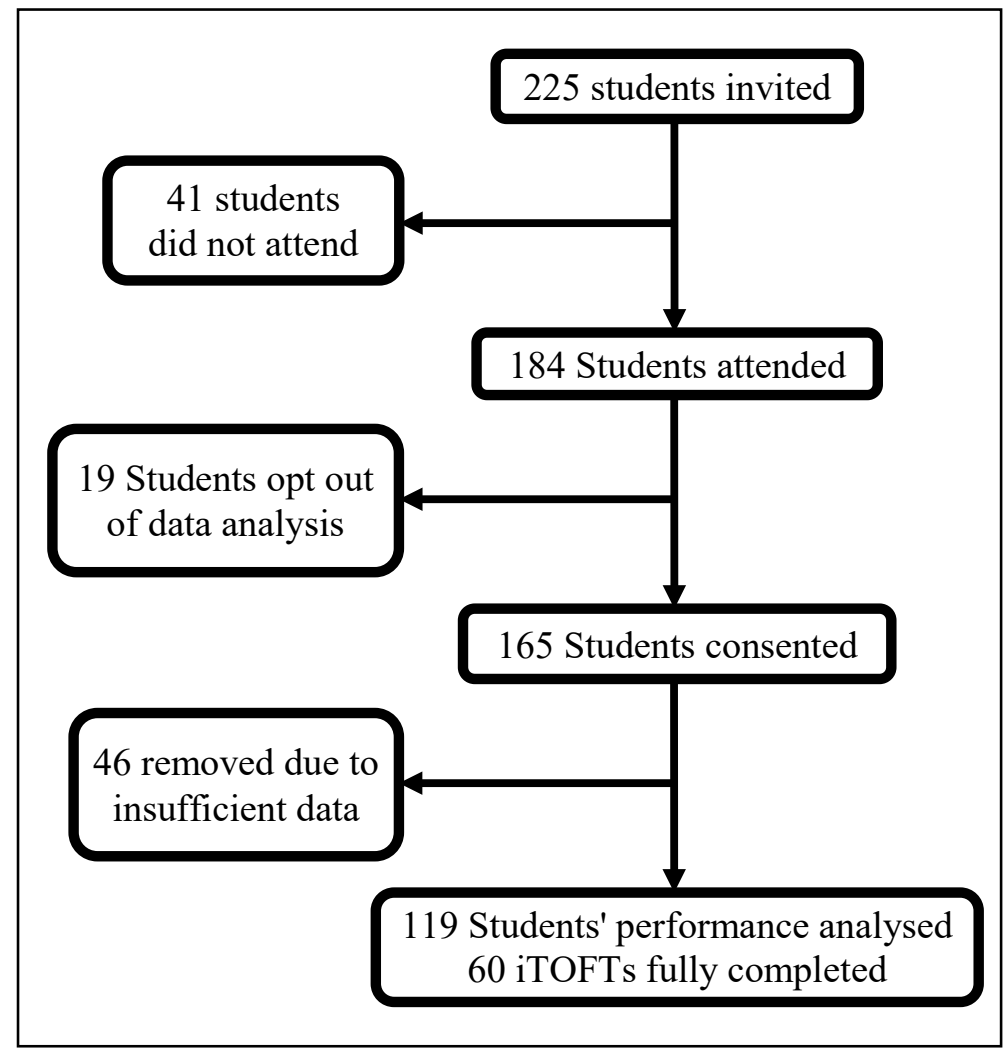

Figure 1: Student recruitment and analysis flow chart

\begin{tabular}{lllll}
\hline Groups & Number of students & Median & IQR & Range \\
\hline Mixed groups & 60 & $11.2^{+}$ & 3.5 & $6.5-13$ \\
Total stratified & 59 & $10.5^{+}$ & 1.625 & $4.5-13$ \\
\hline Top third & 14 & $9.2^{*}$ & 2.79 & $4.7-13$ \\
Mid third & 26 & $11.2^{*}$ & 1.55 & $4.5-13$ \\
Lower third & 19 & $10.5^{*}$ & 0.5 & $10.5-11$ \\
\hline Total & 119 & 11 & 2.5 & $4.5-13$ \\
\hline
\end{tabular}


*p:0.549 Kruskal Wallis, ${ }^{+}$p:0.045 Mann-Whitney

$\mathrm{U}$,

Table 2: Student group sizes and iTOFT scores 